\title{
Three-dimensional structure micelles of heparin- poloxamer improve the therapeutic effect of I7 $\beta$-estradiol on endometrial regeneration for intrauterine adhesions in a rat model
}

\author{
This article was published in the following Dove Press journal: \\ International Journal of Nanomedicine \\ 7 August 2017 \\ Number of times this article has been viewed
}

\author{
Si-Si Zhang ${ }^{1, *}$ \\ Wei-Ting $X_{i a}{ }^{1, *}$ \\ Jie $X u^{2}$ \\ $\mathrm{He}$-Lin $\mathrm{Xu}^{2}$ \\ Cui-Tao Lu ${ }^{2}$ \\ Ying-Zheng Zhao ${ }^{2, *}$ \\ Xue-Qing Wu ${ }^{1, *}$ \\ 'Department of Obstetrics and \\ Gynecology, The First Affiliated \\ Hospital of Wenzhou Medical \\ University, Wenzhou, Zhejiang, \\ People's Republic of China; ${ }^{2}$ School \\ of Pharmaceutical Sciences, Wenzhou \\ Medical University, Wenzhou City, \\ Zhejiang, People's Republic of China \\ *These authors contributed equally \\ to this work
}

Correspondence: Ying-Zheng Zhao School of Pharmaceutical Sciences, Wenzhou Medical University, Wenzhou City, Zhejiang 325035, People's Republic of China Email pharmtds@I63.com

Xue-Qing Wu

Department of Obstetrics and Gynecology, The First Affiliated Hospital of Wenzhou Medical University, Wenzhou City, Zhejiang 325035, People's Republic of China Email Ictuua@sina.com

\begin{abstract}
Intrauterine adhesions (IUA) frequently occur after infectious or mechanical injury to the endometrium, which may lead to infertility and/or pregnancy complications. There are few effective treatments due to the complex function of endometrium and shortage of native materials. $17 \beta$-estradiol $\left(\mathrm{E}_{2}\right)$ is commonly used as an ancillary treatment in IUA patients, but it is limited by its poor solubility in aqueous solutions and low concentrations at the injured sites. In this research, a mini-endometrial curette was used to injure the rat's endometrium to form an IUA model. 17 $\beta$-estradiol was encapsulated into the micelles of heparin-poloxamer and a thermosensitive hydrogel ( $\mathrm{E}_{2}-\mathrm{HP}$ hydrogel) was formed. This sustained releasing system was applied to restore the structure and function of the injured uterus. $\mathrm{E}_{2}$-HP hydrogel was constructed and relevant characteristics including gelation temperature and micromorphology were evaluated. Sustained release of $17 \beta$-estradiol from HP hydrogel was performed both in vitro and in vivo. Ultrasonography measurement and pathologic characteristics on the IUA rats were performed to evaluate the therapeutic effect of $\mathrm{E}_{2}-\mathrm{HP}$ hydrogel. Endoplasmic reticulum (ER) stress-related apoptosis was analyzed to explore the possible mechanisms in IUA recovery. $\mathrm{E}_{2}$-HP hydrogel showed a prolonged release of $\mathrm{E}_{2}$ at the targeting region and more effective endometrium regeneration in IUA rats. Significant improvements in both gland numbers and fibrosis area were observed in the $\mathrm{E}_{2}$-HP hydrogel group. We also demonstrated that $\mathrm{E}_{2}-\mathrm{HP}$ hydrogel in the recovery of IUA was closely related to the suppression of ER stress signals via the activation of downstream signals, PI3K/Akt and ERK1/2. HP hydrogel might be an effective approach to deliver $\mathrm{E}_{2}$ into the injured endometrium. Therapeutic strategies targeting ER stress using $\mathrm{E}_{2}-\mathrm{HP}$ hydrogel might be a promising solution for the treatment of women with intrauterine adhesions.
\end{abstract}

Keywords: intrauterine adhesions, 17ß-estradiol, heparin-poloxamer hydrogel, endometrium regeneration, endoplasmic reticulum stress

\section{Introduction}

Intrauterine adhesions (IUA), also known as Asherman's Syndrome, is characterized by fibrosis or adhesions within the uterine cavity as a result of damage or infection to the basal layer of the endometrium. ${ }^{1}$ IUA increases the risk of menstrual abnormalities, secondary infertility, recurrent miscarriage, and pregnancy complications. ${ }^{2}$ Common treatments include hysteroscopic adhesiolysis and administration of hormonal drugs to promote the regeneration of the endometrial tissue. However, readhesion and side effects frequently occur. ${ }^{3}$ Although hysteroscopic lysis of adhesions is the most 
effective method to re-establish the anatomy of the uterine cavity and restore endometrial function, the procedure itself contributes to readhesions because it may damage the epithelial cells postoperatively. ${ }^{4}$ Therefore, it is imperative to find a treatment that targets the cellular and molecular mechanisms underlying the pathophysiology of IUA.

The endoplasmic reticulum (ER) is an essential intracellular organelle in which proteins are synthesized and folded. Many events such as exposure to free radicals and consumption of ER $\mathrm{Ca}^{2+}$ stores can lead to an unfolded protein response (UPR) and trigger ER stress. ${ }^{5,6}$ Although the original activation of the UPR can protect the cell against these adverse situations, sustained or excessive UPR is harmful and contributes to cell apoptosis. ${ }^{7}$ Accumulation of unfolded proteins within the ER lumen can simultaneously lead to the overexpression of glucose-regulated protein 78 (GRP78/BiP) and $\mathrm{C} / \mathrm{EBP}$ homologous protein (CHOP), and activate the caspase-12, a crucial protein related to cell apoptosis induced by ER stress. ${ }^{8}$ Recent studies have revealed that ER stressinduced apoptosis plays an important role in the pathogenesis and development of many diseases. ${ }^{9}$ Bifulco et al indicated that the activation of UPR and the alteration of GRP78 expression were involved in endometrial cancer development and progression. ${ }^{10}$ However, there are few studies on the role of UPR and ER stress in the treatment of IUA.

$17 \beta$-estradiol $\left(\mathrm{E}_{2}\right)$ is an important steroid hormone. It has been demonstrated that $\mathrm{E}_{2}$ can effectively promote endometrial regeneration and form new capillaries after menstruation. ${ }^{11}$ Estrogen therapy is commonly used as an ancillary treatment after adhesiolysis since it accelerates the regeneration of the endometrial layer and therefore prevents recurrent adhesions. ${ }^{12}$ Evidence from many studies indicates that the application of estrogen therapy in patients with IUA results in satisfactory outcomes. ${ }^{2}$ However, $17 \beta$-estradiol, by either oral delivery or systemic administration, imposes restrictions on its clinical use because of its limited half-life period in vivo, poor solubility in aqueous solutions, and low concentrations at the injured endometrium. Therefore, it may be important to enhance local concentrations to improve the efficacy of $17 \beta$-estradiol.

Heparin-poloxamer (HP), co-polymers consisting of polypropylene oxide (PPO) and polyethylene oxide (PEO) units, have been commonly used as sustained-release drug delivery systems. In response to the alternation of concentration and temperature, the PEO hydrophilic units remain hydrated, while the PPO hydrophobic units are dehydrated and they aggregate, forming the micellar core and corona, respectively. Then, these micelles are self-assembled in ordered cubic or hexagonal phases, producing the thermosensitive hydrogels. They are nontoxic and biodegradable, and with proper formulation, they can increase the solubility, stability, and bioavailability of the incorporated drugs. ${ }^{13}$ In general, HP hydrogel has been widely used for loading and delivering molecules to treat different diseases. ${ }^{13,14}$ Recently, Wu et al developed and characterized an HP hydrogel containing acidic fibroblast growth factor (aFGF) for wound treatments and showed a clear therapeutic effect. ${ }^{15}$ In addition, HP hydrogel loading nerve growth factor (NGF) enhanced the neuron regeneration in spinal cord injury. ${ }^{16}$ Therefore, we consider that HP hydrogel may be a useful drug delivery system for $17 \beta$-estradiol in IUA.

In this research, we attempted to prepare and characterize a novel HP thermosensitive hydrogel loading $17 \beta$-estradiol ( $\mathrm{E}_{2}$-HP hydrogel) with the purpose of developing a sustained releasing delivery system of $17 \beta$-estradiol and further increased its biological effect in vivo. Our results suggested that $\mathrm{E}_{2}$-HP hydrogel had more biological effects than $\mathrm{E}_{2}$ solution only, which showed that the $\mathrm{E}_{2}-\mathrm{HP}$ hydrogel had a therapeutic potential to treat IUA.

\section{Materials and methods Reagents and antibodies}

All reagents used in this study were commercially available. $17 \beta$-estradiol was purchased from Sigma-Aldrich (St Louis, MO, USA). Dulbecco's Modified Eagle's Medium/F-12 (DMEM/F-12) and fetal bovine serum (FBS) were purchased from Invitrogen (Carlsbad, CA, USA). Poloxamer 407-grafted heparin copolymer was synthesized by our laboratory, as previously reported. ${ }^{17}$ GRP78, CHOP, anti-Akt, phosphorylated-Akt (Ser473), anti-ERK, and phosphorylated-ERK antibodies were purchased from Santa Cruz Biotechnology (Santa Cruz, CA, USA). VEGF, Ki67, TGF- $\beta$, and caspase-12 were ordered from Abcam (Abcam, CB, UK). PI3K/Akt inhibitor LY294002, ERK1/2 inhibitor PD98059, and all other reagents were purchased from the Beyotime Institute of Biotechnology (Shanghai, China), unless otherwise specified.

\section{Preparation of $E_{2}-\mathrm{HP}$ hydrogel}

HP hydrogel was prepared according to the 1-ethyl-3-(3dimethylaminopropyl)-carbodiimide (EDC) and N-hydroxylsuccinimide (NHS) method. ${ }^{18} \mathrm{E}_{2}$-HP hydrogel, including different amounts of HP hydrogel and 17 $\beta$-estradiol, was prepared using the cold method. Briefly, lyophilized HP powder was added to purified water under moderate magnetic agitation and in ice bath. The solution was kept at $4^{\circ} \mathrm{C}$ for a period of $24 \mathrm{~h}$ to ensure the complete dissolution of the polymer. Then, $17 \beta$-estradiol was added to a microtube containing $500 \mathrm{~mL}$ of alcohol and solubilized using vortex. The solution was immediately added to the HP solution under vigorous 
magnetic agitation and in an ice bath. The alcohol was completely evaporated under vacuum using a conventional rotary evaporation method (Buchi, Flawil, Switzerland). The final weight of the formulation was set up with purified water to obtain the final concentration of $18 \% \mathrm{w} / \mathrm{w}$ (weight/weight) of HP and $0.2 \mathrm{mg}$ of $17 \beta$-estradiol, per gram of formulation.

\section{Micromorphology of $\mathrm{E}_{2}-\mathrm{HP}$ hydrogel}

The micromorphology of the dehydrated HP hydrogel and $\mathrm{E}_{2}$-HP hydrogel was observed by a scanning electron microscope (SEM; Hitachi, H-7500, Japan). The samples were placed on a copper sheet and dipped into liquid nitrogen immediately. After this process, the hydrogels were dried by a vacuum freeze dryer for $24 \mathrm{~h}$. The dehydrated specimens were then cross-sectioned and sputter-coated with gold. Their surface morphology was observed using an SEM. ${ }^{19}$

\section{In vitro release of drug}

$\mathrm{E}_{2}$ solution and $\mathrm{E}_{2}-\mathrm{HP}$ hydrogel were prepared as previously described. In vitro release of $\mathrm{E}_{2}$ was prepared according to the literature, with some modifications. ${ }^{20}$ In brief, $1 \mathrm{~mL} \mathrm{E}$ solution and $1 \mathrm{~mL} \mathrm{E}_{2}$-HP hydrogel were placed in a tube. Then, a release medium was added to the samples, and the tubes were placed in a shaking incubator at $37^{\circ} \mathrm{C}$ with continuous shaking at $100 \mathrm{rpm}$. At each time point $(0,0.5,1,2,4,8,12$, 24,48 , and $72 \mathrm{~h}$ ), $\mathrm{E}_{2}$ was sampled through the supernatant and then replaced with an equal volume of fresh medium. The released $\mathrm{E}_{2}$ was analyzed by high-performance liquid chromatography at $280 \mathrm{~nm}$. The amount of released $E_{2}$ was normalized through the former and final concentrations. The release experiments were performed in triplicate.

\section{In vivo intrauterine retention and sustained release of drug}

To investigate in vivo intrauterine retention and sustained release of $\mathrm{E}_{2}$-HP hydrogel, $18 \%$ poloxamer hydrogel loaded with $0.2 \mathrm{mg} / \mathrm{mL} 17 \beta$-estradiol-BSA-FITC was prepared and injected into the right cavity of uteri, whereas $17 \beta$-estradiolBSA-FITC solution served as a control group. After injection, rats were sacrificed and the uteri were collected at 1, 2, 8, 24 , and $48 \mathrm{~h}$ followed by washing with saline. Fluorescent images of intact uteri were taken using an In Vivo IVIS spectrum imaging system (a wavelength of $500 \mathrm{~nm}$ and at an excitation wavelength of $465 \mathrm{~nm}$ ).

\section{Isolation and culture of primary mouse endometrial epithelial cells}

The procedure for the isolation of primary mouse endometrial epithelial cells (EECs) was performed according to the literature, but with some modifications. ${ }^{41}$ In brief, the uteri were cut into small pieces and digested with $0.1 \%$ collagenase and $0.25 \%$ trypsin-ethylenediamine-tetra-acetic acid. After digesting for $60 \mathrm{~min}$ under slight shaking, the digestive reaction was stopped by adding the complete medium into solution. Then, the EECs were isolated by the following protocol: the digested cells suspension was centrifuged for $3 \mathrm{~min}$ at $500 \mathrm{rpm}$. After this, DMEM/F-12 culture medium was used to wash the precipitate. Subsequently, trypsinethylenediamine-tetra-acetic acid $(0.25 \%)$ was added to the precipitates and incubated in $\mathrm{CO}_{2}$ at $37^{\circ} \mathrm{C}$ for $10 \mathrm{~min}$. The suspension was centrifuged again at $800 \mathrm{rpm}$ for $5 \mathrm{~min}$ and a complete culture medium was used to resuspend precipitates. The primary EECs were cultured in DMEM/F-12 supplemented with $10 \% \mathrm{FBS}$ and $1 \%$ penicillin and streptomycin in a humidified incubator (Thermo, San Diego, CA, USA) under $5 \% \mathrm{CO}_{2}$ at $37^{\circ} \mathrm{C}$.

\section{Cell viability assay}

EECs were seeded on 96-well plates for $24 \mathrm{~h}$ and treated with different doses of hydrogen peroxide $\left(\mathrm{H}_{2} \mathrm{O}_{2}\right)$ solution. From the cells' survival state, $400 \mu \mathrm{mol} / \mathrm{L} \mathrm{H}_{2} \mathrm{O}_{2}$ was selected for the subsequent experiments. For determining the protection effect of $\mathrm{E}_{2}-\mathrm{HP}$ hydrogel on EECs, various doses of $\mathrm{E}_{2}$ solution and $\mathrm{E}_{2}-\mathrm{HP}$ hydrogel were added after the addition of $\mathrm{H}_{2} \mathrm{O}_{2}$ for different times. Then, $10 \mu \mathrm{L}$ of cell counting kit (CCK)-8 was added into each well. Four hours later, the $450 \mathrm{~nm}$ absorption was observed by a microplate reader.

\section{Animal models and experimental groups}

This study was approved by the Animal Care and Use Committee of Wenzhou Medical University. All animalhandling procedures were performed according to the Guide for the Care and Use of Laboratory Animals from the National Institutes of Health. Female Sprague-Dawley rats (230-250 g) at the age of 8 weeks were purchased from the Animal Center of Chinese Academy of Sciences, Shanghai, China. In this study, the surgical models were created at diestrus stage when the uterine lining began to appear. All the animals were anesthetized by an intraperitoneal injection of $1 \%$ pentobarbital sodium $(0.5 \mathrm{~mL} / \mathrm{kg})$. After this, a vertical incision was made in the abdominal midline and the uteri were identified. A horizontal incision $(0.2 \mathrm{~cm})$ was made on the uterus $0.5 \mathrm{~cm}$ above its bifurcation. The mechanical injury entailed the scraping of endometrium using a miniendometrial curette. Saline, HP hydrogel, $\mathrm{E}_{2}$ solution, and $\mathrm{E}_{2}$-HP hydrogel were injected into bilateral uterine lumens at a dosage of $30 \mu \mathrm{L}$ through the micro syringe immediately after operation. In the sham operation group, animals received 


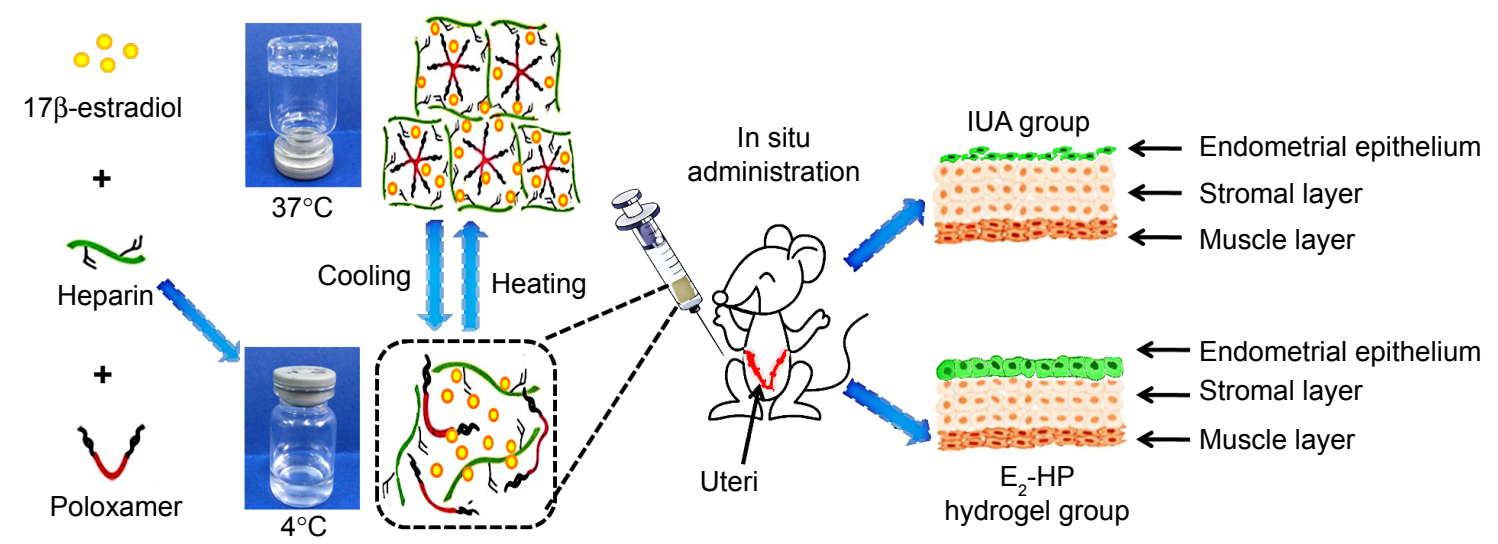

Figure I Schematic diagram of $\mathrm{E}_{2}-\mathrm{HP}$ hydrogel as an in situ administration drug for the treatment of intrauterine adhesions.

Abbreviation: IUA, Intrauterine adhesions.

the same surgical procedures without any treatment for the uteri (Figure 1). According to our preliminary experiments, rats were sacrificed and uteri were harvested at day 14 post surgery.

\section{Ultrasonography measurement}

Fourteen days after surgery, three rats of each group were anesthetized by an intraperitoneal injection of $1 \%$ pentobarbital sodium $(0.5 \mathrm{~mL} / \mathrm{kg})$. The rats were then placed on a cork platform in supine position with preparation of the abdominal skin. Ultrasonography measurements were performed by a trained sonographer. Among all experiments, the standard setting of each parameter was kept consistent.

\section{Histological analysis}

For histological preparation, the uteri were fixed with $4 \%$ paraformaldehyde, embedded in paraffin and sliced into $5 \mu \mathrm{m}$ thickness sections. The transverse sections were stained with hematoxylin-eosin and Masson stains (Beyotime Institute of Biotechnology, Shanghai, China) by conventional methods. The number of glands on each hematoxylin-eosin stained section was counted in each of the five randomly selected fields under high power, and the mean was calculated. Five high-power fields of each Masson-stained section were randomly chosen to quantify the degree of endometrial fibrosis.

\section{Immunohistochemical staining}

For immunohistochemistry, the transverse paraffin sections were deparaffinized, rehydrated, and then incubated in 3\% $\mathrm{H}_{2} \mathrm{O}_{2}$ for $15 \mathrm{~min}$ at room temperature. After this, the sections were heated to recover antigen. Then, the samples were blocked using $5 \%$ bovine serum albumin (BSA) (Beyotime) for $30 \mathrm{~min}$ at $37^{\circ} \mathrm{C}$. Subsequently, the sections were incubated with the following primary antibodies: Ki67 (1:100), VEGF (1:100), TGF- $\beta$ (1:100), CHOP (1:200), GRP78 (1:200), and caspase-12 (1:400) at $4^{\circ} \mathrm{C}$ overnight. Then, the sections were incubated with tagged secondary antibody with either goat antimouse or goat antirabbit for $2 \mathrm{~h}$ at $37^{\circ} \mathrm{C}$. The reaction was stopped with 3,3-diaminobenzidine. The results were imaged using a Nikon ECLPSE 80i (Nikon, Tokyo, Japan). The optical densities and positive staining cell numbers were quantified at five randomly selected fields per section.

\section{Western blot analysis}

Total proteins from injured uterine tissues and primary mouse EECs were purified using protein extraction reagents according to the instructions. The equivalent of $20 \mu \mathrm{g}$ of protein was separated by $12 \%$ gel and transferred onto PVDF membrane (Bio-Rad, Hercules, CA, USA). After blocking with 5\% fatfree milk for $2 \mathrm{~h}$, the membranes were incubated with the relevant protein antibodies overnight. TBST were used to wash the membranes three times. After this process, the membranes were treated with horseradish peroxidase-conjugated secondary antibodies for $1 \mathrm{~h}$ at room temperature. The signals were visualized with the ChemiDicTMXRS + Imaging System (Bio-Rad Laboratories), and the band densities were quantified with Multi Gauge Software of Science Lab 2006 (FUJIFILM Corporation, Tokyo, Japan).

\section{Statistical analysis}

Results were expressed as the mean \pm SD for each group. Student's $t$-tests were performed to determine the significance of 
differences between pairs. One-way analysis of variance was utilized to determine significant differences between multiple groups. $P<0.05$ was considered statistically significant.

\section{Results}

\section{Characterization of $\mathrm{E}_{2}-\mathrm{HP}$ hydrogel}

As shown in Figure 2A, HP hydrogel converted to a gel state quickly after the temperature was heated, while melting to sol at low temperature. This characteristic first allowed $\mathrm{E}_{2}$ to be well blended with HP solution at $4^{\circ} \mathrm{C}$. When delivering the $\mathrm{E}_{2}$-HP solution to the damaged endometrium in situ at $37^{\circ} \mathrm{C}$, HP formed hydrogel, allowing $\mathrm{E}_{2}$ to be successfully loaded into HP hydrogel.

From the observation of SEM, there was little difference in micromorphology of HP hydrogels in the presence or absence of loaded $\mathrm{E}_{2}$, demonstrating that the encapsulation of $\mathrm{E}_{2}$ in HP hydrogel did not affect its structure (Figure 2B1 and B2). Both blank hydrogel and $\mathrm{E}_{2}$-HP hydrogel showed a threedimensional structure similar to that of the porous sponge, in which the inner pores interconnected with each other. This structure allowed $\mathrm{E}_{2}$ to diffuse through these pores and control its release rate.

\section{In vitro release of estradiol}

As supporting data for the in vivo application, we tested in vitro estradiol release from the in situ gel-forming system at $37^{\circ} \mathrm{C}$ (Figure 3 ). $\mathrm{E}_{2}$ solution $(1 \mathrm{mg} / \mathrm{mL}$ ) served as the control, for which almost all drug $(\sim 80 \%)$ was immediately released to dissolve the medium at $2 \mathrm{~h}$ in the release study. However, $\mathrm{E}_{2}$-HP hydrogel demonstrated a sustained release of $\mathrm{E}_{2}$ for over $72 \mathrm{~h}$. At the end of the experiment, about $95 \% \mathrm{E}_{2}$ was released from the hydrogel. These results further suggested that the structure of the gel functioned as a resistant barrier to the release of $\mathrm{E}_{2}$ at body temperature. Prolonged release of $\mathrm{E}_{2}$ at a constant rate from the hydrogel might make it a useful biomaterial for the long-term treatment of IUA.

\section{In vivo intrauterine retention and release of estradiol}

As shown in Figure 4, 17ß-estradiol-BSA-FITC revealed sustained release from the hydrogel after infusion into the right uterus. Over time, the fluorescence intensity in the uterine cavity gradually became weaker. At $48 \mathrm{~h}$, the fluorescence signals in the uterus completely disappeared. Compared with the HP hydrogel, the fluorescence intensity of $17 \beta$-estradiol-BSA-FITC solution decreased rapidly and almost disappeared at $2 \mathrm{~h}$. These results proved the controlled-release behavior of HP hydrogel for the loaded micromolecule, which provided the foundation for the application of $\mathrm{E}_{2}-\mathrm{HP}$ hydrogel in IUA treatment.

\section{$\mathrm{E}_{2}-\mathrm{HP}$ hydrogel promotes EEC survival rate in vitro}

To investigate an appropriate concentration of $\mathrm{H}_{2} \mathrm{O}_{2}$ and $\mathrm{E}_{2}$ in EECs, we conducted dose-response experiments. We found
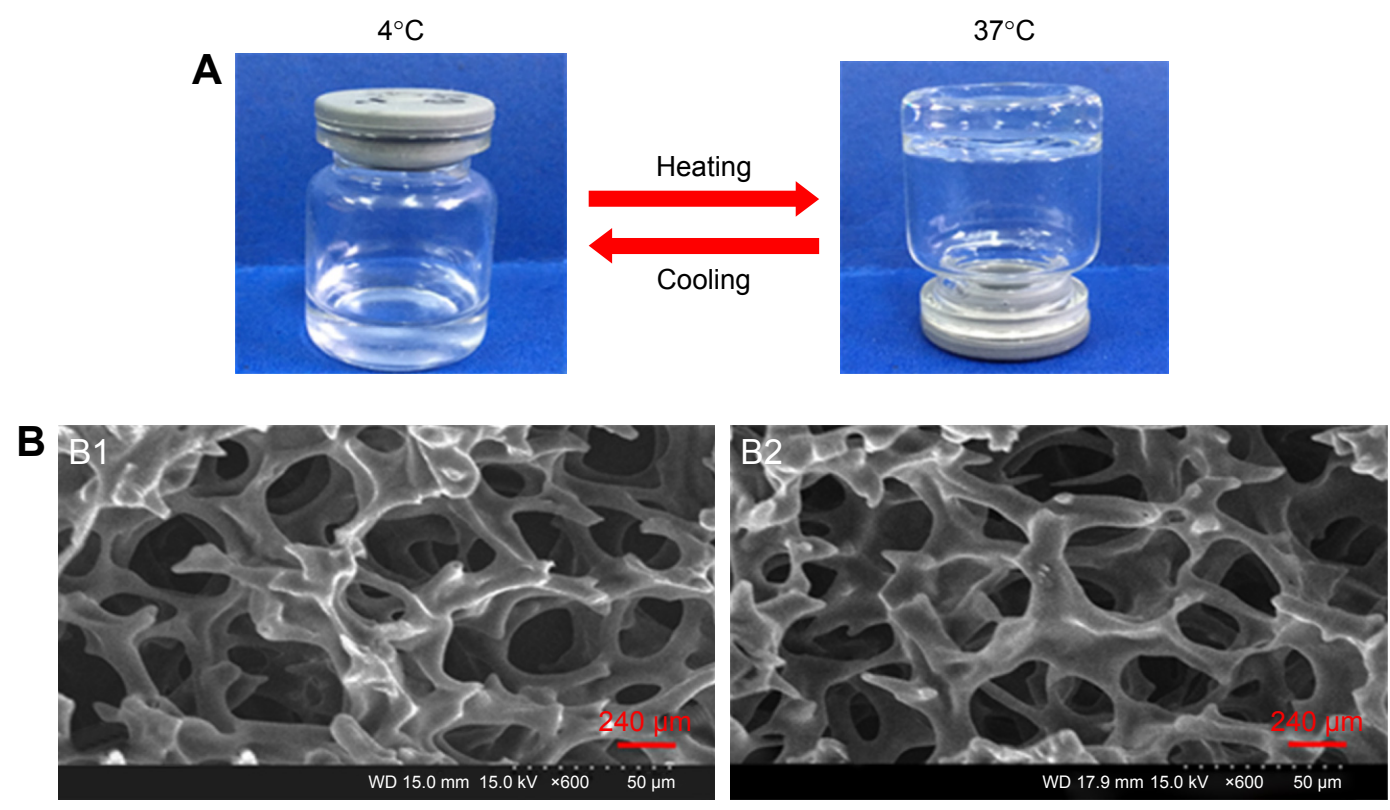

Figure 2 Characteristics of $E_{2}-\mathrm{HP}$ hydrogel.

Notes: (A) The state of $\mathrm{E}_{2}-\mathrm{HP}$ hydrogel at different temperatures $\left(4^{\circ} \mathrm{C}\right.$ and $\left.37^{\circ} \mathrm{C}\right)$. (B) SEM images of the morphology of $\mathrm{HP}$ hydrogel $(\mathrm{BI})$ and $\mathrm{E}_{2}-\mathrm{HP}$ hydrogel (B2). Scale bars in BI and B2 are $240 \mu \mathrm{m}$.

Abbreviation: SEM, scanning electron microscope. 


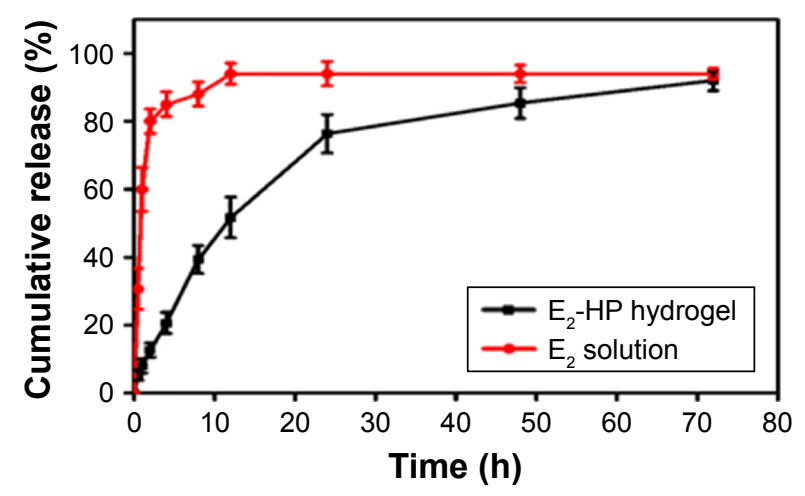

Figure 3 The cumulative release of $E_{2}$ in vitro.

Notes: Different time points are 0.5, I, 2, 4, 8, 12, 24, 48, and 72 h. Data are presented as mean \pm standard deviation; $n=5$.

that incubation with $\mathrm{H}_{2} \mathrm{O}_{2}$ led to dose-dependent cell death after a 4-hour exposure. From the result, $400 \mu \mathrm{mol} / \mathrm{L} \mathrm{H}_{2} \mathrm{O}_{2}$ was chosen to establish the cell model. In addition, the cell viability of EECs after treatment with $\mathrm{E}_{2}$ solution increased significantly at the concentrations of $100 \mathrm{nmol} / \mathrm{L}$ in all tested incubation times (Figure 5A). On the basis of this result, $100 \mathrm{nmol} / \mathrm{L} \mathrm{E}_{2}$ solution was selected for subsequent experiments. As shown in Figure 5B, remarkable proliferation of EECs was noted after treatment with $\mathrm{E}_{2}$ solution or $\mathrm{E}_{2}-\mathrm{HP}$ hydrogel for 24,48 , and $72 \mathrm{~h}$. $\mathrm{E}_{2}$-HP hydrogel group was obviously higher than that in $\mathrm{E}_{2}$ solution. No significant difference was found between control and HP hydrogel groups. These results further suggested that the occurrence of a sustained-release of $E_{2}$ from the $E_{2}-H P$ hydrogel might be profitable for cell proliferation.

\section{Ultrasonography measurement of the injured uterus in vivo}

In this study, we assessed the treatment outcomes of IUA using two-dimensional ultrasound. In the sham operation group, the echo of endometrium was uniform with a clearly visible endometrial line in the middle. However, an uneven internal echo of the uterine cavity was seen in IUA and HP hydrogel groups, and the endometrial line was discontinuous in these groups. After treatment with $\mathrm{E}_{2}$ solution, this line was slightly restored in the damaged uterus. Treating with $\mathrm{E}_{2}-\mathrm{HP}$ hydrogel showed a better repairing effect than $\mathrm{E}_{2}$ solution group (Figure 6). These results convincingly demonstrated that the administration of $\mathrm{E}_{2}$-HP hydrogel improved the regeneration of endometrium on IUA.

\section{$E_{2}-H P$ hydrogel increases the number of glands and decreases the fibrosis area in IUA model}

Hematoxylin-eosin staining results of the uteri samples from the different experimental groups after 14 days were shown in Figure 7A. In the sham operation group, the endometrial surface was lined with simple high columnar epithelial cells. Round or oval glands were primarily found in the submucosa and basal layer, and there were large openings
$1 \mathrm{~h}$
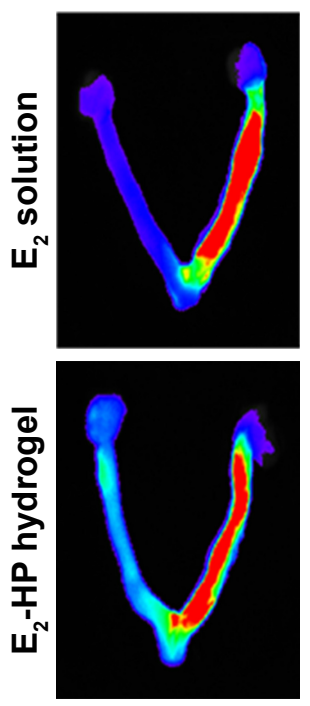

$2 \mathrm{~h}$
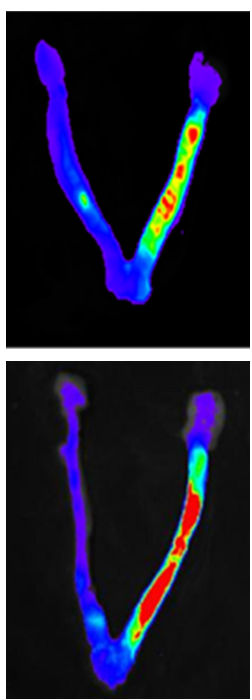

$8 \mathrm{~h}$
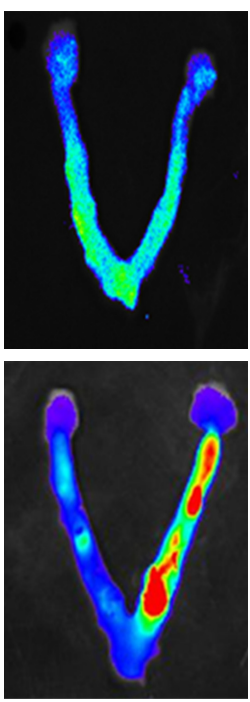

$24 \mathrm{~h}$
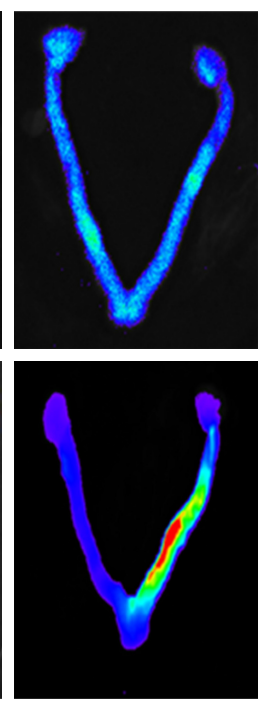

$48 \mathrm{~h}$

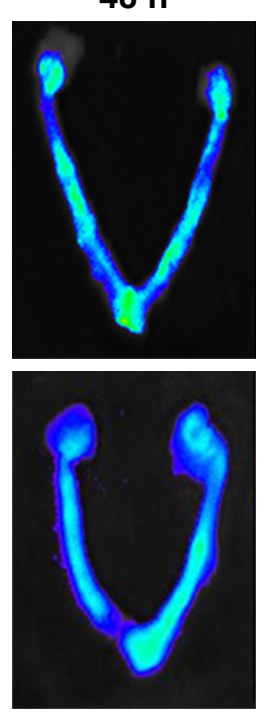

Epi-fluorescence

0.8

0.6

$\times 10^{9}$

$-0.4$

0.2

Radiant efficiency, luminous flux per unit area $\left(\frac{\mathrm{P} / \mathrm{sec} / \mathrm{cm}^{2} / \mu}{\mu \mathrm{w} / \mathrm{cm}^{2}}\right)$

Color scale Min $=6.13 \mathrm{e} 7$ Max $=9.83 \mathrm{e} 8$

Figure 4 The penetration and retention of different FITC- $E_{2}$ formulations in the uterus after in situ administration. Notes: Different time points are I, 2, 8, 24, and 48 h; $n=5$. 

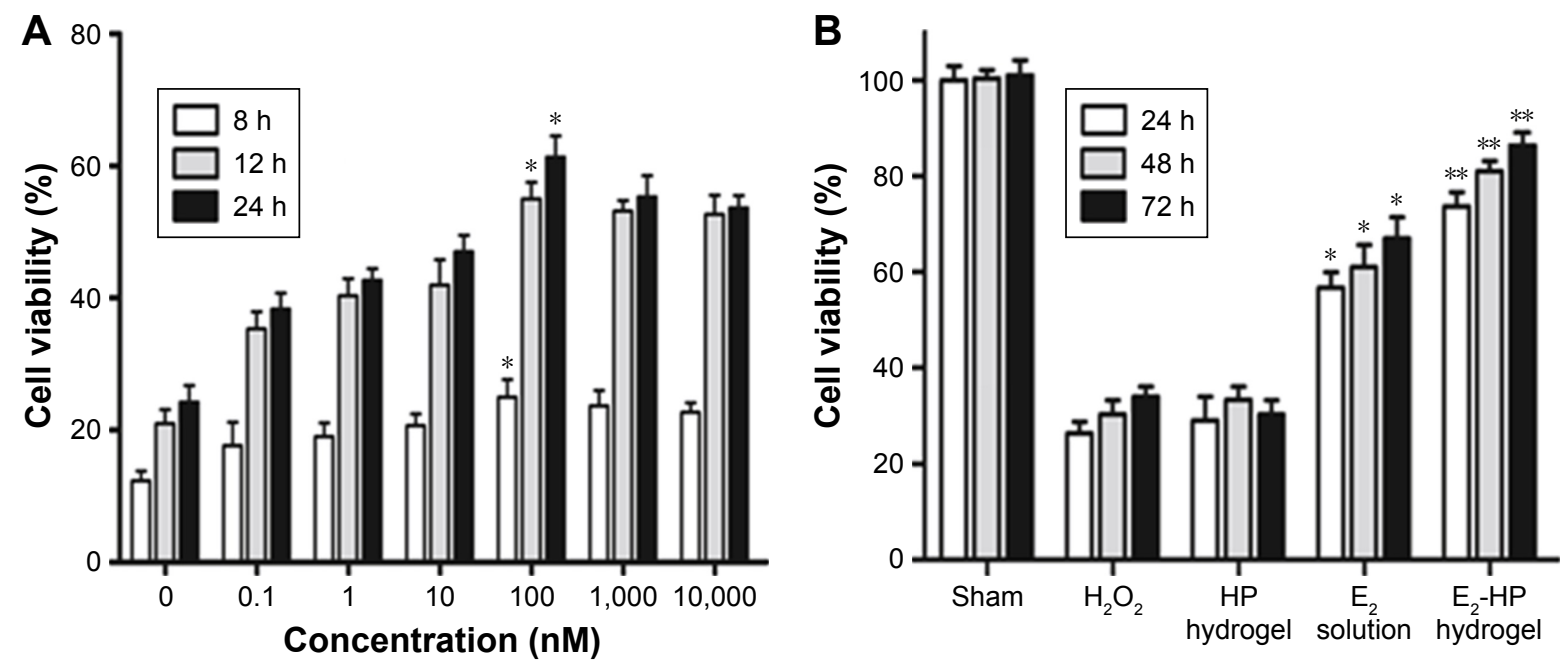

Figure 5 Cell viability of various groups in vitro.

Notes: (A) Endometrial epithelial cells (EECs) were pretreated with $\mathrm{H}_{2} \mathrm{O}_{2}$ for $4 \mathrm{~h}$ and were treated with different concentrations of $\mathrm{E}_{2}$ solution for $8 \mathrm{~h}, 12 \mathrm{~h}$, and $24 \mathrm{~h}$. Then, the cell viability was assessed by the CCK-8 assay. $* P<0.05$ versus $\mathrm{H}_{2} \mathrm{O}_{2}$ group. (B) EECs were pretreated with $\mathrm{H}_{2} \mathrm{O}_{2}$ for $4 \mathrm{~h}$ and were treated with different treatments for 24 , 48 , and $72 \mathrm{~h}$. Then, the cell viability was assessed by the CCK-8 assay. $* P<0.05$ and $* * P<0.01$ versus $\mathrm{H}_{2} \mathrm{O}_{2}$ group. Data are presented as mean \pm standard deviation; $\mathrm{n}=3$. Abbreviations: CCK-8, cell counting kit-8; EECs, endometrial epithelial cells.

at the endometrial surface (Figure 7A). Fourteen days after endometrial scraping, the surface in the IUA group was almost covered by flat and low columnar epithelial cells, and adhesion of uterine cavity could be detected in some animals. Meanwhile, a few glands were visible beneath the epithelial layer (Figure 7A). No difference was found in the number of glands between IUA group and HP hydrogel group. Compared with the IUA group, the number of glands on day 14 after intervention slightly increased in the $\mathrm{E}_{2}$ solution group.
The $\mathrm{E}_{2}$-HP hydrogel group showed remarkably higher numbers of glands, similar to the sham operation group and better than the $\mathrm{E}_{2}$ solution group (Figure 7). These results indicated that synergistic interactions between $\mathrm{HP}$ hydrogel and $\mathrm{E}_{2}$ offered a more efficient repair effect at the endometrial injury site.

To evaluate the fibrosis of damaged endometrium in the IUA model, Masson staining of collagen was performed to measure the ratios of the endometrial fibrosis area (Figure 7B and D). In the sham operation group, there was
Sham

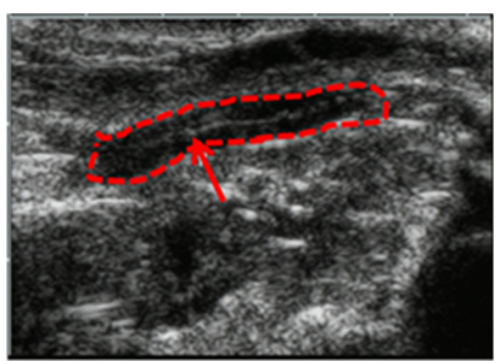

IUA

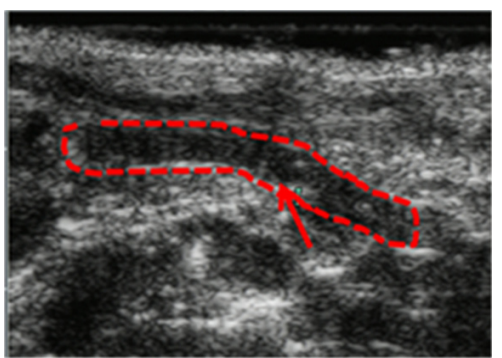

HP hydrogel

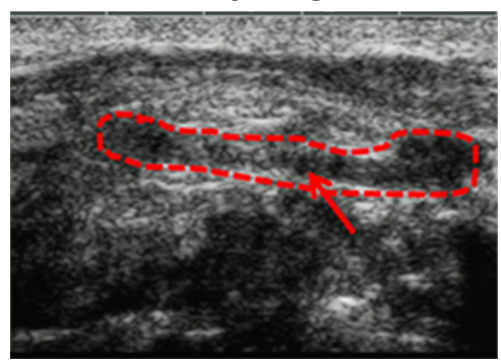

$E_{2}-$ HP hydrogel

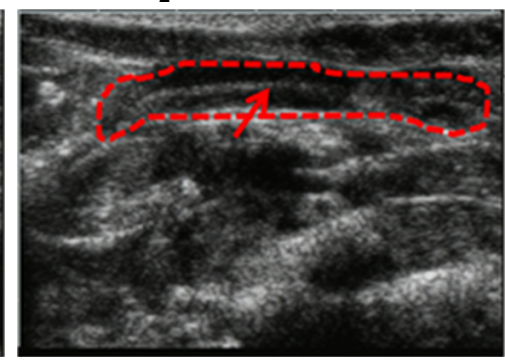

Figure 6 Ultrasonography measurement of the injured uterus in different groups at 14 days after IUA.

Notes: The red dotted lines indicate the uterine cavity. The red arrowheads indicate the endometrial line; $n=5$.

Abbreviations: IUA, Intrauterine adhesions; HP, heparin-poloxamer. 
A
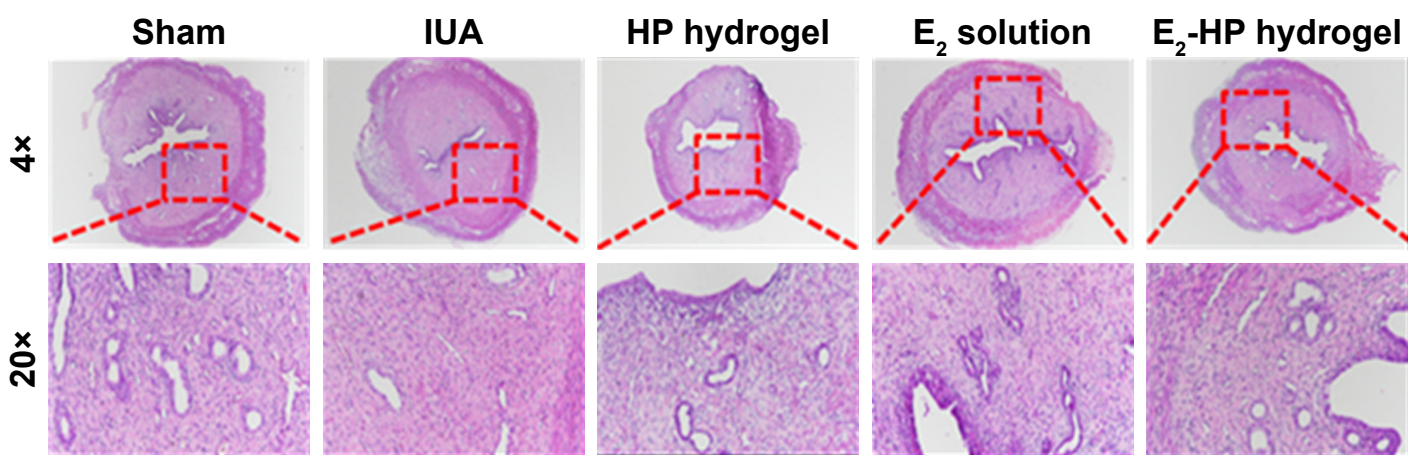

B
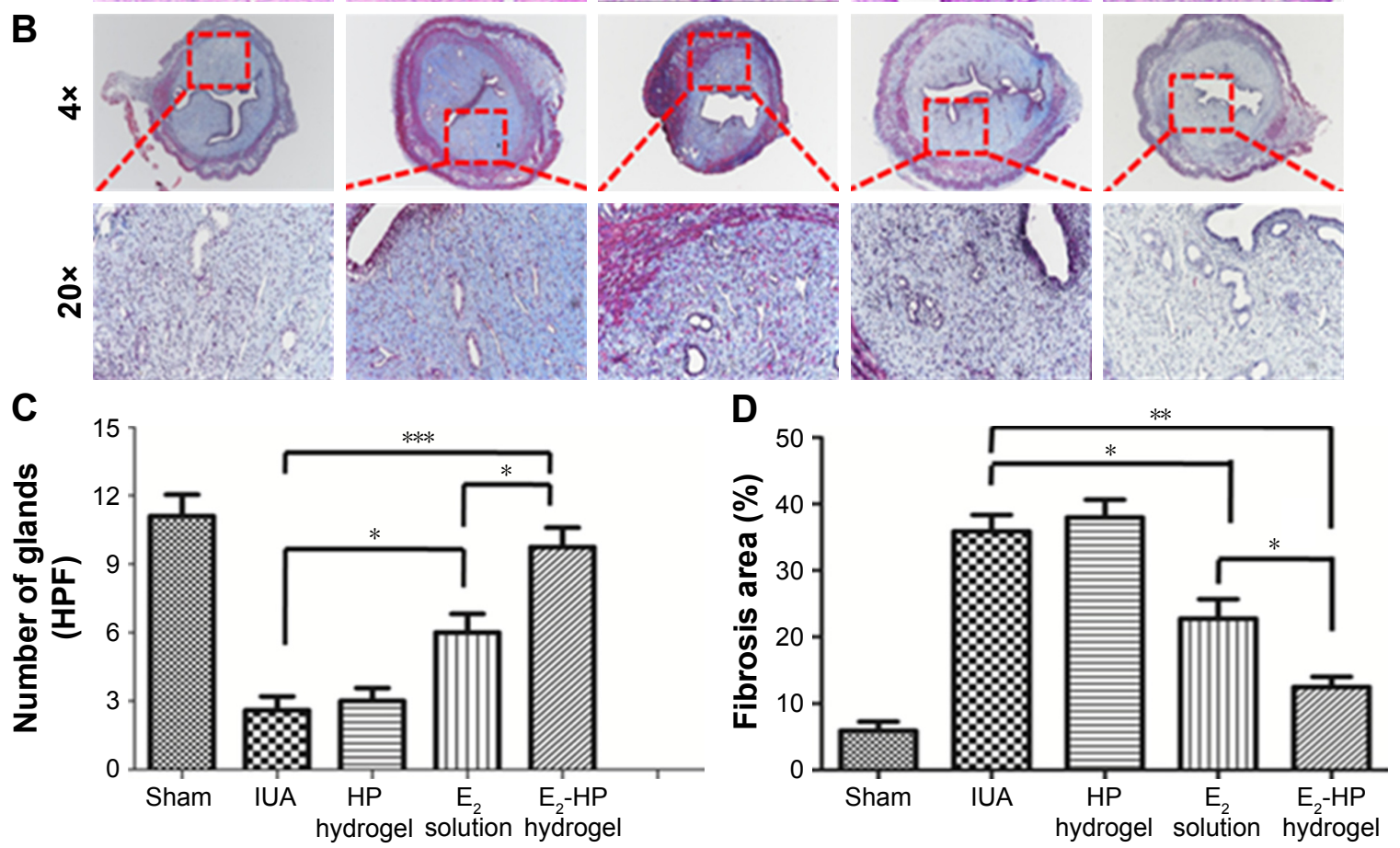

Figure 7 Histological structures of the injured uterus in different groups.

Notes: (A) HE staining of crosscutting results $(40 \times$ and $200 \times)$ at I 4 days after IUA. (B) Masson's trichrome method stain of the collagen (40× and $200 \times)$ at I4 days after IUA. (C) Analysis of the number of glands in each group at 14 days after IUA. (D) Analysis of fibrosis area in endometrium in each group at 14 days after IUA. Data are presented as mean \pm standard deviation; $\mathrm{n}=5 ; * \mathrm{p}<0.05, * * \mathrm{p}<0.0 \mathrm{I}$, and $* * * \mathrm{p}<0.00 \mathrm{I}$.

Abbreviations: IUA, Intrauterine adhesions; HE, haematoxylin-eosin staining; HP, heparin-poloxamer.

almost no collagen deposition in the endometrial stroma. In contrast, the ratio of the area with endometrial fibrosis in the IUA group was significantly increased compared with the sham operation group. There was no significant difference in the extent of endometrial fibrosis between the IUA and HP hydrogel groups. Treatment with $\mathrm{E}_{2}$ solution alone did not obviously reduce the endometrial fibrosis in IUA rats. Only when the IUA rats were subjected to the combination treatment of $\mathrm{E}_{2}$-HP hydrogel, the degree of endometrial fibrosis was moderately but significantly decreased. As shown in Figure 7, the change in endometrial fibrosis was inversely related to the number of glands in the rats of each group.

\section{$\mathrm{E}_{2}-\mathrm{HP}$ hydrogel promotes cell proliferation and vascularization in the injured endometrium}

To evaluate the effect of $\mathrm{E}_{2}$-HP hydrogel treatment on the regeneration of endometrium, we assessed the expression of Ki67, a nuclear antigen for cell proliferation at all stages, to assess whether $\mathrm{E}_{2}$-HP hydrogel could promote the proliferation of the endometrial cell. As shown in Figure 8A, the numbers of proliferative cells were weakly detected or were absent in the IUA group. No significant difference in Ki67 expression was observed between IUA and HP hydrogel groups. Compared with the IUA group, we demonstrated a statistically significant increase of Ki67 at the epithelial compartment 
A

A
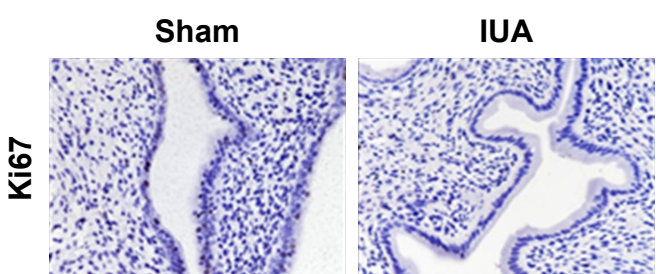

HP hydrogel
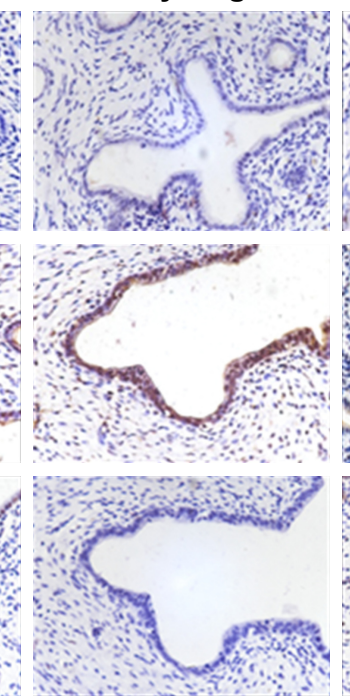

B
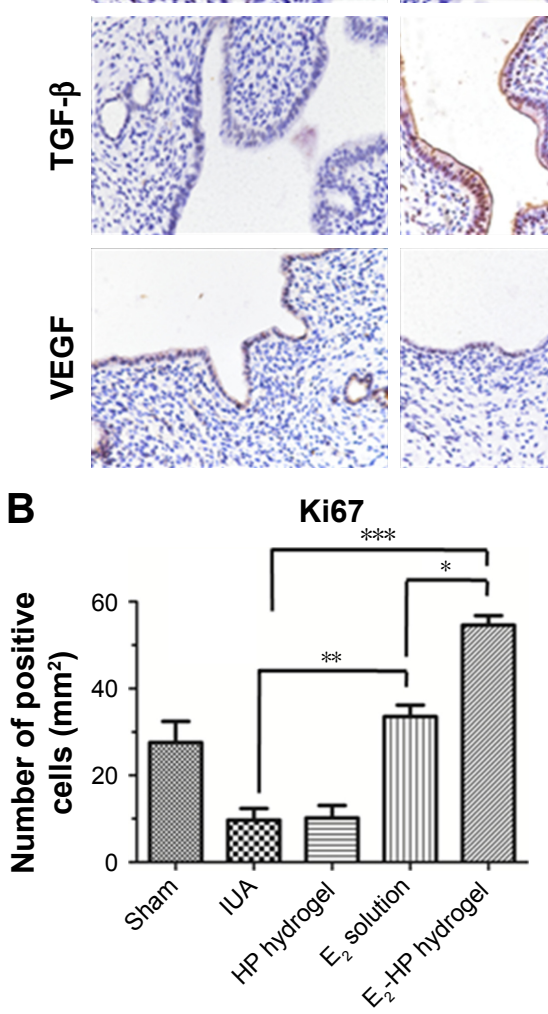

TGF- $\beta$

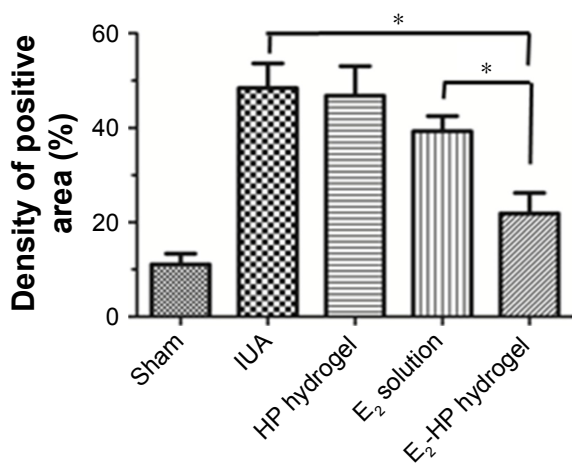

$E_{2}$ solution
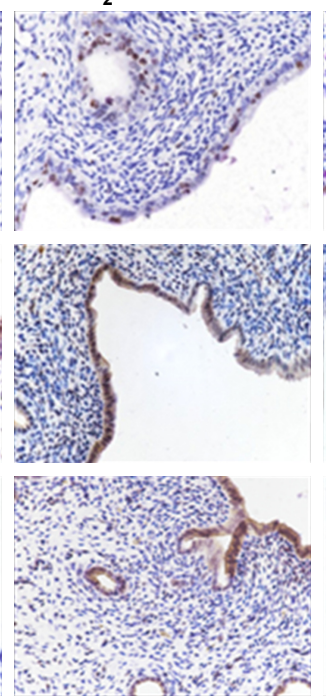

D
$E_{2}-$ HP hydrogel
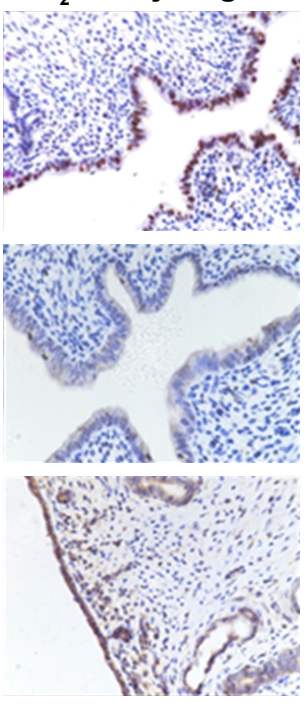

VEGF

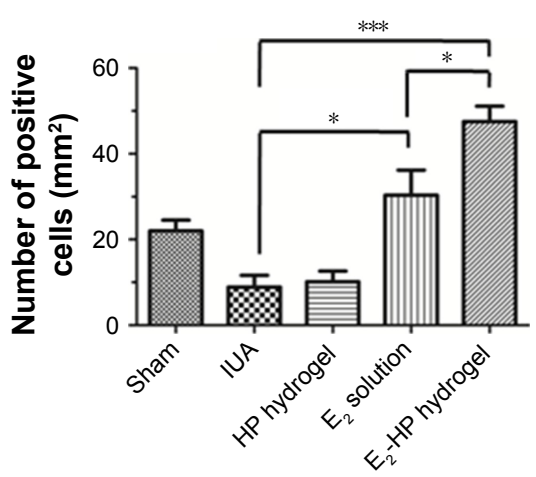

Figure 8 Immunohistochemical staining of Ki67, TGF- $\beta$, and VEGF.

Notes: (A) The expression of Ki67, TGF- $\beta$, and VEGF in the immunohistochemical staining of endometrium (400 $\times$ ) on the I4th day after IUA with different treatments. (B) Analysis of Ki67 positive cells of the immunohistochemistry results. (C) Analysis of TGF- $\beta$ positive area of the immunohistochemistry results. (D) Analysis of VEGFpositive cells of the immunohistochemistry results. Data are presented as mean \pm standard deviation; $n=5 ; * P<0.05, * * P<0.0$ I, and $* * * P<0.00$ I.

Abbreviations: IUA, Intrauterine adhesions; HP, heparin-poloxamer.

in the $\mathrm{E}_{2}-\mathrm{HP}$ hydrogel group. The $\mathrm{E}_{2}$ solution alone also improved the regeneration of IUA, but $\mathrm{E}_{2}$-HP hydrogel led to a more remarkable improvement of injury. These results buttressed the fact that the administration of $\mathrm{E}_{2}$-HP hydrogel improved the proliferation of endometrium on IUA.

Immunohistochemical staining demonstrated that TGF- $\beta$ was mainly expressed both in the nucleus and in the cytoplasm of epithelial and stromal cells. As shown in Figure 8A, the expression of TGF- $\beta$ in the endometrium of IUA was distinctly increased compared with that in the sham operation group. Moreover, TGF- $\beta$ showed strong expression in the endometrial tissue of HP hydrogel group. The quantity of TGF- $\beta$ in the $\mathrm{E}_{2}$ solution group was mildly lower than that of the IUA group. Most strikingly, we observed that the level of TGF- $\beta$ significantly declined in the $\mathrm{E}_{2}-\mathrm{HP}$ hydrogel group, similar to that in the sham operation group (Figure 8C). Collectively, these results indicated that TGF- $\beta$ may play a crucial role in IUA formation. And delivering of $\mathrm{E}_{2}$ with the thermosensitive heparin poloxamer hydrogel was effective in inhibiting the expression of TGF- $\beta$.

Vascular endothelial growth factor (VEGF) is a wellknown angiogenic factor, which is essential for neovascularization. Evidence shows that VEGF also has additional functions such as antiapoptosis and vascular remodeling. To explore the possible involvement of VEGF in the repair of endometrium, we estimated the quantity of VEGF by performing immunohistochemical staining (Figure 8A and D). The results showed that VEGF was significantly decreased in IUA and HP hydrogel groups compared with that in the sham operation group. After the administration of $\mathrm{E}_{2}$ solution, 

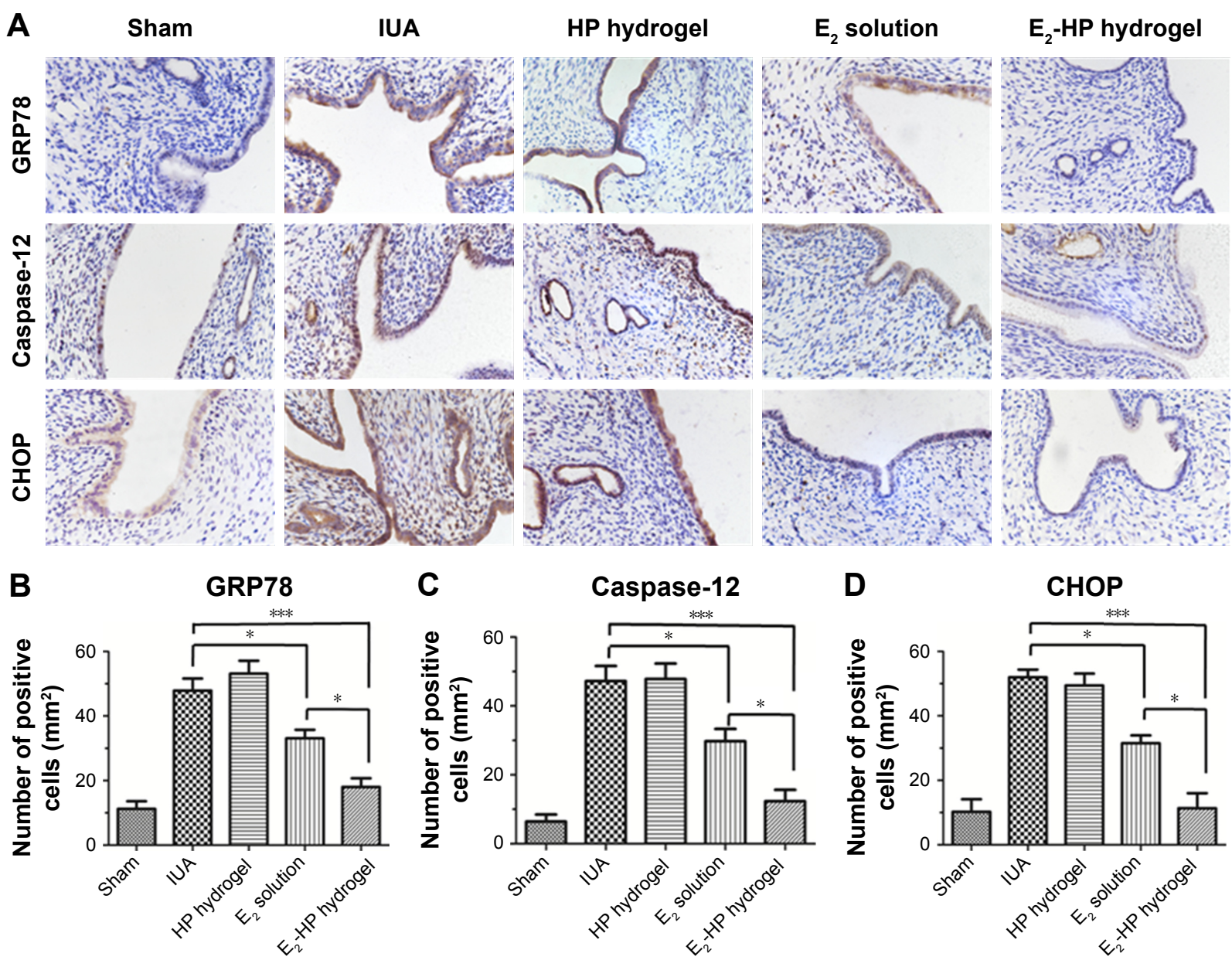

Figure $9 \mathrm{E}_{2}$-HP hydrogel inhibits ER stress-induced apoptosis in IUA rats.

Notes: (A) Immunohistochemistry for GRP78, caspase-12, and CHOP (400x) on the 14th day after different treatments with IUA; (B) Quantitative analysis of GRP78positive cells of the immunohistochemistry results. (C) Quantitative analysis of caspase-12-positive cells of the immunohistochemistry results. (D) Quantitative analysis of CHOP-positive cells of the immunohistochemistry results. Data are presented as mean \pm standard deviation; $\mathrm{n}=5 ; * \mathrm{P}<0.05$ and $* * * \mathrm{P}<0.00 \mathrm{I}$.

Abbreviations: CHOP, C/EBP homologous protein; ER, endoplasmic reticulum; GRP78, glucose-regulated protein; IUA, Intrauterine adhesions; HP, heparin-poloxamer.

the level of VEGF was slightly upregulated in the damaged endometrium, whereas treating with $\mathrm{E}_{2}-\mathrm{HP}$ hydrogel showed more evident expression of VEGF than the $\mathrm{E}_{2}$ solution group. To further validate the expression of VEGF in endometrium, a Western blot assay was performed, with a representative blot exhibited in Figure 10A. The relative expression of VEGF proteins was counted through normalization to GAPDH, as summarized in Figure 10C. Consistent with the results of immunohistochemical staining, changes in VEGF expression were observed across experimental groups. Taken together, these findings indicated that HP hydrogel enhanced the therapeutic effect of $E_{2}$ on angiopoiesis after IUA.

\section{$E_{2}-H P$ hydrogel inhibits ER stress signaling pathway in IUA model}

To further explore whether the mechanism of $\mathrm{E}_{2}$-HP hydrogel is related to the alternation of ER stress, the expression of the
ER stress-induced apoptosis signal pathway was evaluated. As shown in Figure 9, ER stress-related apoptosis protein (GRP78, caspase-12 and CHOP) positive cells were obviously increased in the IUA group compared with those in the sham operation group. No significant difference in apoptosis protein expression was observed between IUA and HP hydrogel groups. Meanwhile, positive cells reduced in the $\mathrm{E}_{2}$ solution and $\mathrm{E}_{2}$-HP hydrogel groups. It was evident that $\mathrm{E}_{2}$-HP hydrogel resulted in less positive cells than $\mathrm{E}_{2}$ solution alone. Furthermore, the expression of GRP78, CHOP, and cleaved caspase- 12 were also analyzed by Western blot analysis (Figure 10A and B). Similar to the results of immunohistochemical staining, the levels of the ER stress-related protein were significantly elevated in the injured region of the endometrium in IUA group when compared with the sham operation group. Treatment with $\mathrm{E}_{2}$ solution suppressed the activation of ER stress-induced proteins, and these protein 
A

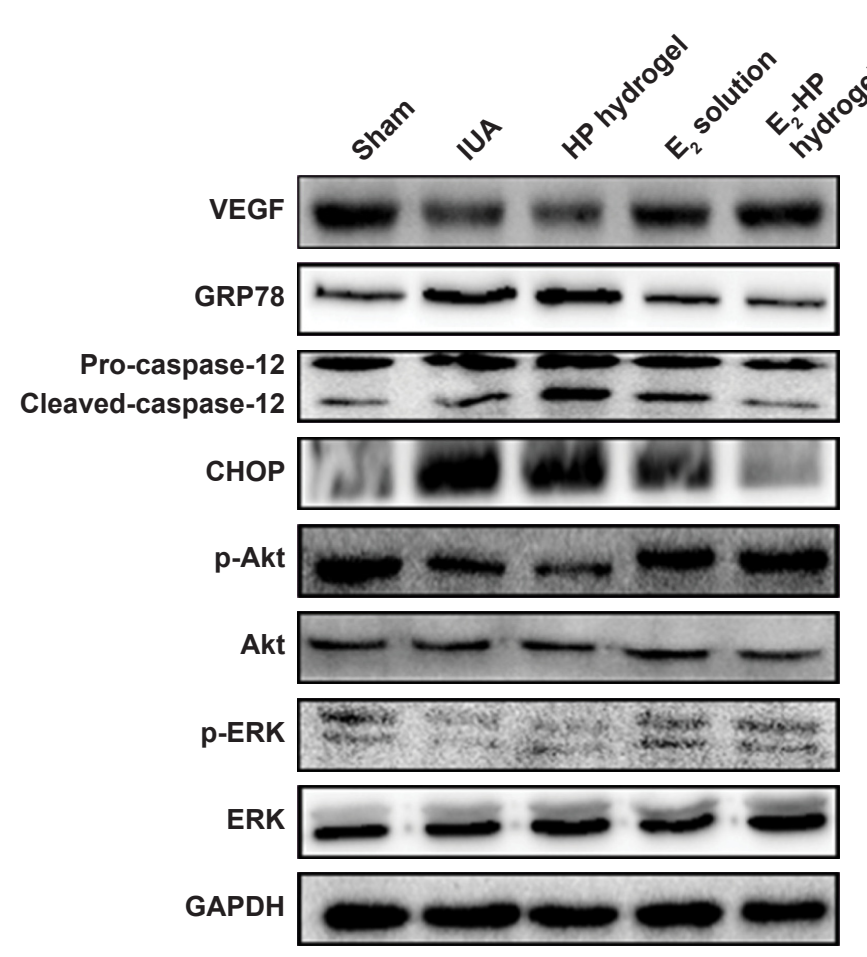

B

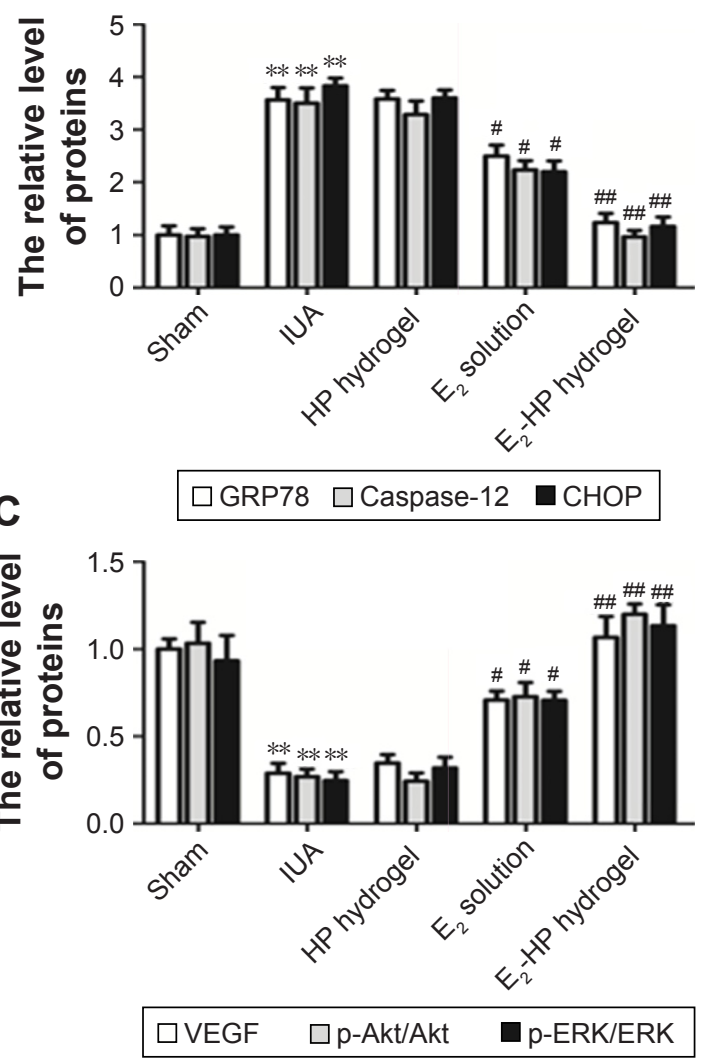

Figure $10 \mathrm{E}_{2}-\mathrm{HP}$ hydrogel inhibits ER stress and activates the Akt and ERKI/2 pathways in the IUA rats.

Notes: (A) The protein expressions of VEGF, GRP78, caspase-12, CHOP, p-Akt, and p-ERK in each group were tested with Western blotting. GAPDH was used as the loading control and for band density normalization. (B) The optical density analysis of GRP78, caspase- 12 , and $C H O P$ protein. $* * P<0.01$ versus the Sham group and $\# P<0.05$ and ${ }^{\#} P<0.0$ I versus the IUA group. (C) The optical density analysis of VEGF, $p$-Akt, and $p$-ERK protein. ${ }^{* * P}<0.0$ I versus the Sham group and ${ }^{\# P}<0.05$ and ${ }^{\#} P<0.0$ I versus the IUA group. Data are presented as mean \pm standard deviation; $n=3$.

Abbreviations: CHOP, C/EBP homologous protein; ER, endoplasmic reticulum; GRP78, glucose-regulated protein; IUA, Intrauterine adhesions; HP, heparin-poloxamer.

levels in $\mathrm{E}_{2}$-HP hydrogel-treated rats were relatively lower than those in the $\mathrm{E}_{2}$ solution group.

\section{The protective role of $\mathrm{E}_{2}-\mathrm{HP}$ hydrogel is related to the activation of downstream signal pathways $\mathrm{PI} 3 \mathrm{~K} / \mathrm{Akt}$ and ERKI/2}

PI3K/Akt and ERK1/2 pathways are two main downstream signals activated by $\mathrm{E}_{2}$. These signal pathways are strongly linked to cell survival, migration, and differentiation. To further explore the underlying mechanisms behind the effect of $\mathrm{E}_{2}$-HP hydrogel in the recovery of IUA, the activation of PI3K/Akt and ERK1/2 downstream signals was evaluated. Our Western blot analysis revealed that the levels of phosphorylation of Akt and ERK1/2 decreased after IUA intervention. However, the reductions of p-Akt and $p$-ERK1/2 were reversed after the administration of $E_{2}$ solution or $\mathrm{E}_{2}$-HP hydrogel. The $\mathrm{E}_{2}$-HP hydrogel group showed more obvious upregulation of these proteins than did the $E_{2}$ solution group (Figure 10A and C). Taken together, these results suggested that the protective role of $\mathrm{E}_{2}-\mathrm{HP}$ hydrogel in endometrial impairment was linked to the inhibition of ER stress through the activation of the PI3K/Akt and ERK1/2 signaling pathways.

\section{The activation of downstream signals is vital for the protective effect of $E_{2}-H P$ hydrogel}

To further explore the molecular mechanism of $\mathrm{E}_{2}-\mathrm{HP}$, the PI3K inhibitor LY294002 and the ERK1/2 inhibitor PD98,059 were added to the media. In our previous study, none of these inhibitors showed any effect on cell death. As shown in Figure 10A, $\mathrm{H}_{2} \mathrm{O}_{2}$-induced activation of GPR78, cleaved-caspase-12, and CHOP was decreased after treatment with $\mathrm{E}_{2}-\mathrm{HP}$ hydrogel. After the combined exposure to Akt/ ERK1/2 inhibitors, the inhibitory effect of $\mathrm{E}_{2}-\mathrm{HP}$ hydrogel on the expression of GRP78, cleaved caspase-12, and CHOP 


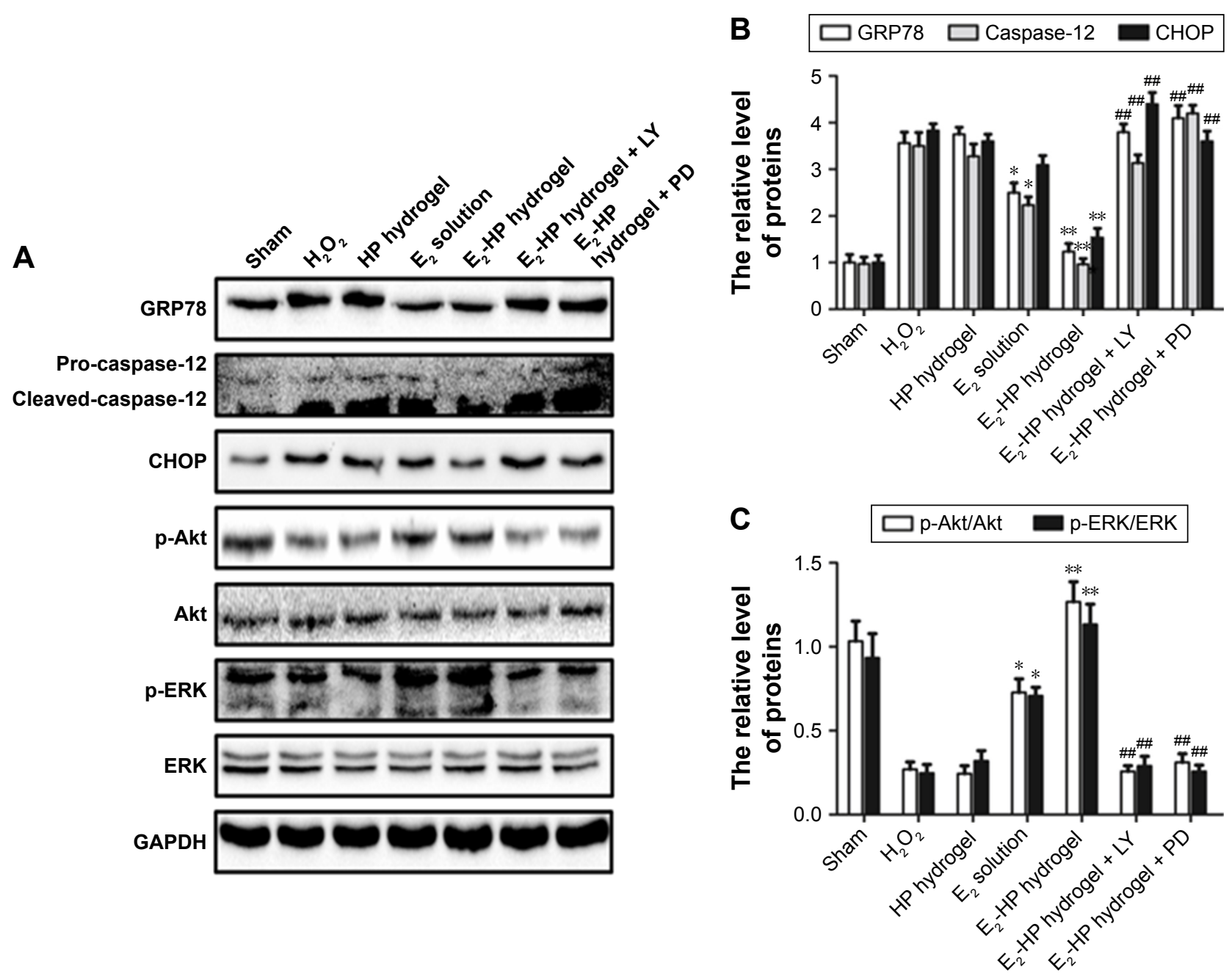

Figure II The activation of Akt and ERKI/2 is crucial for the protective effect of $E_{2}$-HP hydrogel in $\mathrm{H}_{2} \mathrm{O}_{2}$-induced ER stress in EECs.

Notes: (A) The protein expressions of GRP78, caspase-12, CHOP, p-Akt, and p-ERKI/2 in ER stress-induced apoptosis in EECs treated with $E_{2}-H P$ hydrogel and different inhibitors. GAPDH was used as the loading control and for band density normalization. (B) The optical density analysis of GRP78, caspase- I2, and CHOP protein. (C) The optical density analysis of $p$-AKT and $p$-ERK protein. ${ }^{*} P<0.05$ and ${ }^{*} * P<0.01$ versus the $\mathrm{H}_{2} \mathrm{O}_{2}$ group and ${ }^{\#} P<0.01$ versus the $E_{2}$-HP hydrogel group. Data are presented as mean \pm standard deviation; $\mathrm{n}=3$.

Abbreviations: CHOP, C/EBP homologous protein; EECs, endometrial epithelial cells; ER, endoplasmic reticulum; GRP78, glucose-regulated protein; HP, heparin-poloxamer.

was reversed (Figure 11A and B). Consistent with this, our Western blot results demonstrated that $\mathrm{E}_{2}-\mathrm{HP}$ hydrogel triggered the activation of p-Akt, and p-ERK1/2 was also attenuated by Akt/ERK1/2 inhibitors (Figure 11A and C). All of these results suggested that the protective effect of $\mathrm{E}_{2}-\mathrm{HP}$ hydrogel was regulated by both the PI3K/Akt and ERK1/2 signaling pathways.

\section{Discussion}

Intrauterine adhesions usually occur as a result of infectious or mechanical injury to the endometrium, leading to partial or complete adhesions of the wall of the uterine cavity and/or the cervical canal. Ever since Heinrich Fritsch first described this condition in a patient with secondary amenorrhea, the incidence of IUA has seen an increase in some parts of the world. $^{21}$ In China, it has been reported that about $7 \%$ of cases of secondary amenorrhea and $40 \%$ of cases of infertility are caused by IUA, which remains a severe threat to reproductive women. ${ }^{2}$ Over the decades, many experimental studies have focused on the regeneration of endometrium and on the prevention of scar reformation. One of the traditional therapeutic approaches for intrauterine adhesions is placing an IUD in the uterine cavity. However, because of their poor biocompatibility, IUDs can impede normal endometrial regeneration and easily cause infection. ${ }^{21,22}$ At present, some possible treatments of IUA include direct delivery of specific growth factors or stem cells into the uterus. A recent study by Zhao et al showed a positive effect of bone marrow mesenchymal stem cells treatment on a rat model with thin endometrium. ${ }^{23}$ Moreover, a collagen scaffold loaded with 
collagen-binding human basic fibroblast growth factor was employed to facilitate the recovery of rat uterine horns, and this delivery system accelerated the regenerative abilities of uterus and attained a better pregnancy outcome. ${ }^{24}$ But all of these treatments available to moderate and severe IUA are limited and inefficient. Thus, more studies and new therapies are urgently needed to better understand the pathogenesis of this disease.

$17 \beta$-estradiol is an essential member of the steroid hormones. It is responsible for uterine endometrial regeneration through stimulating the proliferation and differentiation of stem cells after menstruation. In clinical practice, postoperative treatment with estrogen therapy is considered as the most effective method to re-establish a normal uterine cavity and restore endometrium function in IUA patients. ${ }^{25}$ A recent systematic review provided sufficient evidence that the administration of estrogen could reduce reformation of IUA by promoting endometrial repair and covering the denuded endometrial layer. ${ }^{2}$ Moreover, many studies revealed that using estrogen therapy alone as ancillary treatment was associated with satisfactory outcomes. The restoration of menstrual flow and pregnancy rate was substantially increased when compared with the group that did not receive any hormone therapy. ${ }^{26,27}$ Although these are promising results, there are still many limitations that need to be addressed. First, recurrence of uterine cavity adhesion after estrogen therapy is frequently observed, especially in severe IUA patients. ${ }^{28}$ Second, the $17 \beta$-estradiol at high systemic levels may increase the risk for thrombosis and malignancy. ${ }^{29}$ Third, by either oral delivery or systemic administration, $17 \beta$-estradiol always shows a low concentration at the injured uterus, which may reduce its therapeutic effect.

Based on these situations, in situ administration of $17 \beta$ estradiol may be a suitable delivery choice. However, it is hard to retain $17 \beta$-estradiol solution at the injury endometrium through simple injections or infusions because of its rapid diffusion into extracellular fluids. ${ }^{20}$ Therefore, the aim of this study is to develop a new sustained releasing system intended for the in situ administration of $17 \beta$-estradiol in the uterus and characterized by extended local exposure, fewer side effects, and lower production cost.

HP hydrogel, which has good biocompatibility and a controlled phase alteration temperature, is appropriate for in situ administration. ${ }^{15,30}$ In a typical experiment, HP hydrogel exhibited a liquid state in low temperature. After it was locally administered in vivo, a three-dimensional network structure was formed which sustained the drug releasing from the HP hydrogel. In our previous study, HP hydrogel loaded with growth factors was synthesized and it showed remarkable therapeutic outcomes in wound healing. ${ }^{16} \mathrm{We}$ also found that HP hydrogel effectively increased the delivery of NGF via the blood-spinal cord barrier and showed a significant neuroprotective improvement in rats with spinal cord injury. ${ }^{14}$ Therefore, a similar delivery system was applied in this study. We provided the first evidence that $17 \beta$-estradiol-loaded HP hydrogel effectively enhanced endometrial regeneration and the IUA recovery compared with $17 \beta$-estradiol only in cellular and animal models.

In this work, $\mathrm{E}_{2}-\mathrm{HP}$ hydrogel showed a prolonged release of $\mathrm{E}_{2}$ at the targeting region and more effective endometrium regeneration at 14 days after surgery than did all the other groups. The number of glands was significantly increased in the $\mathrm{E}_{2}$-HP hydrogel group, similar to that in the sham operation group. In addition, the ratio of fibrotic tissue area was markedly decreased after treatment with $\mathrm{E}_{2}-\mathrm{HP}$ hydrogel. Moreover, there was an apparent increase in the number of proliferative cells in the $\mathrm{E}_{2}$-HP hydrogel group than in the control group. Furthermore, in this study, the in situ administration of $\mathrm{E}_{2}-\mathrm{HP}$ hydrogel increased the expression of angiogenesis factor, VEGF, in the damaged area, suggesting that angiogenesis might improve in the treated area. Thus, the $\mathrm{E}_{2}$-HP hydrogel-induced regeneration of endometrium might result from increased vascularity and therefore increased access to nutrients, oxygen, and hormones in the damaged area.

In our study, we not only showed the effectiveness of $\mathrm{E}_{2}$-HP hydrogel on IUA, but also explored the possible mechanisms underlying the therapeutic effects of $E_{2}-\mathrm{HP}$ hydrogel. There is increasing evidence to suggest that ER stress signal plays an important role in promoting cell death in many diseases. A recent study demonstrated that ER stress-induced apoptosis was related to the response of spinal cord injury and that the expression of apoptosis-related proteins, including GRP78, caspase-12, and CHOP protein, was obviously increased after injury. ${ }^{31} \mathrm{~A}$ similar phenomenon was observed in myocardial ischemia/reperfusion injury. ${ }^{32}$ However, the role of ER stress signaling in IUA has never been reported. In the current study, we first showed that ER stress-induced apoptosis was involved in the progression of IUA. The levels of related proteins increased evidently in vivo, and was confirmed in EECs' injury models induced by $\mathrm{H}_{2} \mathrm{O}_{2}$. On the basis of these studies, suppressing this process and protecting IUA-associated apoptosis may be a novel therapeutic strategy for IUA.

Moreover, Haas et al had indicated that dextrose-induced ER stress in human umbilical vein endothelial cells was 
reversed by $\mathrm{E}_{2} \cdot{ }^{33}$ Another study published by Guo et al revealed that $17 \beta-\mathrm{E}_{2}$ protected MC3T3-E1 cells against ER stress-induced apoptosis by accelerating Ras-ERK1/2-TFII-I signaling pathway-dependent GRP78 induction. ${ }^{34}$ In this study, we revealed that exogenous $\mathrm{E}_{2}$-HP hydrogel treatment after injury reduced the expressions of ER stress-induced apoptosis proteins and promoted the regeneration of endometrium. In addition, the repair activity of $\mathrm{E}_{2}-\mathrm{HP}$ hydrogel was also revealed in the $\mathrm{H}_{2} \mathrm{O}_{2}$-induced EECs' injury model. Taken together, these results indicated that ER stress-induced apoptosis could be inhibited by exogenous $\mathrm{E}_{2}$-HP hydrogel administration.

As the main downstream signals activated by $E_{2}$, the $\mathrm{PI} 3 \mathrm{~K} / \mathrm{Akt}$ and ERK1/2 pathways play an important role in several cellular processes, including proliferation, survival, and motility. ${ }^{35,36} \mathrm{~A}$ recent study demonstrated that estrogen in low dose was closely related to rapid increase in Akt and ERK phosphorylation in rat hippocampus, thus promoting cell survival of the neurons. ${ }^{37}$ Moreover, Zhou et al had reported that $17 \beta$-estradiol-induced keratinocyte proliferation might be mediated by the Akt/Erk signaling pathway. ${ }^{38} \mathrm{In}$ addition, a previous study had shown that $\mathrm{E}_{2}$ was involved in the migration of endometrial stromal cells by activating the same signaling routes. ${ }^{39}$ Besides these findings, a previous in vitro study illustrated that low concentrations of $\mathrm{E}_{2}$ were able to counteract endoplasmic reticulum stress-induced apoptosis by Akt pathway in SGC7901 cells. ${ }^{40}$ Therefore, we hypothesized that $\mathrm{E}_{2}$ accelerated the regeneration of injured endometrium by suppressing ER stress-induced apoptosis via the ERK/Akt signaling pathway. In our study, the levels of phosphorylation Akt and phosphorylation ERK significantly declined both in vivo and in vitro. Moreover, the reductions of p-Akt and p-ERK1/2 were reversed after treatment with $\mathrm{E}_{2}$-HP hydrogel. To further certify that these two pathways were vital for the protective effect of $\mathrm{E}_{2}$-HP hydrogel, the PI3K/Akt inhibitor LY294002 and the ERK1/2 inhibitor PD98059 were used to treat EEC cells. Results revealed that apoptosis induced by $\mathrm{H}_{2} \mathrm{O}_{2}$ was inhibited by treating with $\mathrm{E}_{2}$-HP hydrogel and further vanished by this combination of inhibitors. Taken together, we speculated that the role of $\mathrm{E}_{2}$-HP hydrogel in IUA recovery was involved in the activation of both the PI3K/Akt and ERK1/2 signals.

In conclusion, the current study demonstrated that the administration of $\mathrm{E}_{2}$-HP hydrogel was effective in IUA caused by endometrial damage in the rat model. This was related to the inhibiting of ER stress-induced apoptosis and augmenting of the angiogenesis factor VEGF. Furthermore, activation of the two downstream signaling pathways PI3K/
Akt and ERK1/2 was indispensable for the effect of $E_{2}-H P$ hydrogel in endometrial cell regeneration both in vivo and in vitro. However, the complex mechanism of endometrium regeneration in the IUA model needs to be further investigated before an in situ administration technique can be brought from bench to bedside. Since the goal of this research is to reveal safety and short-term efficacy of this drug delivery system, more studies for the long term effect of single versus repeated treatments using this approach are needed. Moreover, alternate approaches, such as to deliver $\mathrm{E}_{2}$ combined with growth factors to the injury, may also be promising strategies.

\section{Acknowledgments}

This research was supported by the Science and Technology Project of Zhejiang Province (Grant No 2016C37132), National Natural Science Foundation of China (Grant No 81571392 and 81603036), Zhejiang Provincial Program for the Cultivation of High-level Innovative Health Talents (Ying-Zheng Zhao), 151 Talent Project of Zhejiang Province and 551 Talent Project of Wenzhou (Ying-Zheng Zhao), 551 Talent Project of Wenzhou (Xue-Qing Wu), Zhejiang Provincial Foundation for Health Department (Grant No 2015ZDA023 and 2016KYA136), key support of highlevel talent innovation and technology project of Wenzhou (Ying-Zheng Zhao), medicine grant from the Wenzhou Bureau of Science and Technology (Grant No Y2014730 and Y20140726), and the Public Welfare Scientific andTechnology Project of Wenzhou (No Y20140743).

\section{Disclosure}

The authors report no conflicts of interest in this work.

\section{References}

1. Hooker AB, Lemmers M, Thurkow AL, et al. Systematic review and meta-analysis of intrauterine adhesions after miscarriage: prevalence, risk factors and long-term reproductive outcome. Hum Reprod Update. 2014;20(2):262-278.

2. Johary J, Xue M, Zhu X, Xu D, Velu PP. Efficacy of estrogen therapy in patients with intrauterine adhesions: systematic review. J Minim Invasive Gynecol. 2014;21(1):44-54.

3. Gilman AR, Dewar KM, Rhone SA, Fluker MR. Intrauterine adhesions following miscarriage: look and learn. J Obstetr Gynaecol Can. 2016;38(5):453-457.

4. Di Spiezio Sardo A, Calagna G, Scognamiglio M, O’Donovan P, Campo R, De Wilde RL. Prevention of intrauterine post-surgical adhesions in hysteroscopy. A systematic review. Eur J Obstet Gynecol Reprod Biol. 2016;203:182-192.

5. Zhang HY, Wang ZG, Lu XH, et al. Endoplasmic reticulum stress: relevance and therapeutics in central nervous system diseases. Mol Neurobiol. 2015;51(3):1343-1352.

6. Pluquet $\mathrm{O}$, Pourtier A, Abbadie C. The unfolded protein response and cellular senescence. A review in the theme: cellular mechanisms of endoplasmic reticulum stress signaling in health and disease. Am J Physiol Cell Physiol. 2015;308(6):C415-C425. 
7. Battson ML, Lee DM, Gentile CL. Endoplasmic reticulum stress and the development of endothelial dysfunction. Am J Physiol Heart Circ Physiol. 2017;312(3):H355-H367.

8. Ohri SS, Maddie MA, Zhao Y, Qiu MS, Hetman M, Whittemore SR. Attenuating the endoplasmic reticulum stress response improves functional recovery after spinal cord injury. Glia. 2011;59(10):1489-1502.

9. Rosas C, Orostica L, Poblete C, et al. Hyperandrogenism decreases GRP78 protein level and glucose uptake in human endometrial stromal cells. Reprod Sci. 2016;23(6):761-770.

10. Bifulco G, Miele C, Di Jeso B, Beguinot F, Nappi C, Di Carlo C, et al. Endoplasmic reticulum stress is activated in endometrial adenocarcinoma. Gynecol Oncol. 2012;125(1):220-225.

11. Seyedoshohadaei F, Tangestani L, Zandvakili F, Rashadmanesh N. Comparison of the effect of clomiphene-estradiol valerate vs letrozole on endometrial thickness, abortion and pregnancy rate in infertile women with polycystic ovarian syndrome. J Clin Diagn Res. 2016;10(8): QC10-QC13.

12. AAGL Advancing Minimally Invasive Gynecology Worldwide. AAGL practice report: practice guidelines for management of intrauterine synechiae. J Minim Invasive Gynecol. 2010;17(1):1-7.

13. Akkari AC, Papini JZ, Garcia GK, et al. Poloxamer 407/188 binary thermosensitive hydrogels as delivery systems for infiltrative local anesthesia: physico-chemical characterization and pharmacological evaluation. Mater Sci Eng C Mater Biol Appl. 2016;68: 299-307.

14. Wang X, Wang J, Wu W, Li H. Vaginal delivery of carboplatin-loaded thermosensitive hydrogel to prevent local cervical cancer recurrence in mice. Drug Deliv. 2016;23(9):3544-3551.

15. Wu J, Zhu J, He C, et al. Comparative study of heparin-poloxamer hydrogel modified $\mathrm{bFGF}$ and aFGF for in vivo wound healing efficiency. ACS Appl Mater Interfaces. 2016;8(29):18710-18721.

16. Zhao YZ, Jiang X, Xiao J, et al. Using NGF heparin-poloxamer thermosensitive hydrogels to enhance the nerve regeneration for spinal cord injury. Acta Biomater. 2016;29:71-80.

17. Zhao YZ, Lv HF, Lu CT, et al. Evaluation of a novel thermosensitive heparin-poloxamer hydrogel for improving vascular anastomosis quality and safety in a rabbit model. PLoS One. 2013;8(8):e73178.

18. Yoo MK, Cho KY, Song HH, et al. Release of ciprofloxacin from chondroitin 6-sulfate-graft-poloxamer hydrogel in vitro for ophthalmic drug delivery. Drug Dev Ind Pharm. 2005;31(4-5):455-463.

19. Niu G, Zhang H, Song L, et al. Thiol/acrylate-modified PEO-PPOPEO triblocks used as reactive and thermosensitive copolymers. Biomacromolecules. 2008;9(10):2621-2628.

20. Cespi M, Bonacucina G, Pucciarelli S, et al. Evaluation of thermosensitive poloxamer 407 gel systems for the sustained release of estradiol in a fish model. Eur J Pharm Biopharm. 2014;88(3):954-961.

21. Evans-Hoeker EA, Young SL. Endometrial receptivity and intrauterine adhesive disease. Semin Reprod Med. 2014;32(5):392-401.

22. Chen Y, Chang Y, Yao S. Role of angiogenesis in endometrial repair of patients with severe intrauterine adhesion. Int J Clin Exp Pathol. 2013;6(7):1343-1350.

23. Zhao J, Zhang Q, Wang Y, Li Y. Uterine infusion with bone marrow mesenchymal stem cells improves endometrium thickness in a rat model of thin endometrium. Reprod Sci. 2015;22(2):181-188.

24. Li X, Sun H, Lin N, et al. Regeneration of uterine horns in rats by collagen scaffolds loaded with collagen-binding human basic fibroblast growth factor. Biomaterials. 2011;32(32):8172-8181.

International Journal of Nanomedicine

\section{Publish your work in this journal}

The International Journal of Nanomedicine is an international, peerreviewed journal focusing on the application of nanotechnology in diagnostics, therapeutics, and drug delivery systems throughout the biomedical field. This journal is indexed on PubMed Central, MedLine, CAS, SciSearch $®$, Current Contents $\AA /$ Clinical Medicine,
25. Liu AZ, Zhao HG, Gao Y, Liu M, Guo BZ. Effectiveness of estrogen treatment before transcervical resection of adhesions on moderate and severe uterine adhesion patients. Gynecol Endocrinol. 2016;32(9): 737-740.

26. Cai H, Li H, He Y. Interceed and Estrogen Reduce Uterine Adhesions and Fibrosis and Improve Endometrial Receptivity in a Rabbit Model of Intrauterine Adhesions. Reprod Sci. 2016;23(9):1208-1216.

27. Dawood A, Al-Talib A, Tulandi T. Predisposing factors and treatment outcome of different stages of intrauterine adhesions. J Obstet gynaecol Can. 2010;32(8):767-770.

28. Malhotra N, Bahadur A, Kalaivani M, Mittal S. Changes in endometrial receptivity in women with Asherman's syndrome undergoing hysteroscopic adhesiolysis. Arch Gynecol Obstet. 2012;286(2):525-530.

29. Zhu L, Jiang X, Sun Y, Shu W. Effect of hormone therapy on the risk of bone fractures: a systematic review and meta-analysis of randomized controlled trials. Menopause. 2016;23(4):461-470.

30. Liu D, Jiang T, Cai W, et al. An in situ gelling drug delivery system for improved recovery after spinal cord injury. Adv Healthc Mater. 2016; 5(12):1513-1521.

31. Zhu SP, Wang ZG, Zhao YZ, et al. Gelatin nanostructured lipid carriers incorporating nerve growth factor inhibit endoplasmic reticulum stress-induced apoptosis and improve recovery in spinal cord injury. Mol Neurobiol. 2016;53(7):4375-4386.

32. Wang Z, Wang Y, Ye J, et al. bFGF attenuates endoplasmic reticulum stress and mitochondrial injury on myocardial ischaemia/reperfusion via activation of PI3K/Akt/ERK1/2 pathway. J Cell Mol Med. 2015;19(3):595-607.

33. Haas MJ, Raheja P, Jaimungal S, Sheikh-Ali M, Mooradian AD. Estrogen-dependent inhibition of dextrose-induced endoplasmic reticulum stress and superoxide generation in endothelial cells. Free Radic Biol Med. 2012;52(11-12):2161-2167.

34. Guo YS, Sun Z, Ma J, et al. 17beta-Estradiol inhibits ER stress-induced apoptosis through promotion of TFII-I-dependent Grp78 induction in osteoblasts. Lab Invest. 2014;94(8):906-916.

35. Peng S, Zhang Y, Zhang J, Wang H, Ren B. ERK in learning and memory: a review of recent research. Int J Mol Sci. 2010;11(1):222-232.

36. Matsumura S, Ohta T, Yamanouchi K, et al. Activation of estrogen receptor alpha by estradiol and cisplatin induces platinum-resistance in ovarian cancer cells. Cancer Biol Ther. Epub 2016 Sep 30.

37. Pratap UP, Patil A, Sharma HR, et al. Estrogen-induced neuroprotective and anti-inflammatory effects are dependent on the brain areas of middle-aged female rats. Brain Res Bull. 2016;124:238-253.

38. Zhou T, Yang Z, Chen Y, et al. Estrogen accelerates cutaneous wound healing by promoting proliferation of epidermal keratinocytes via Erk/Akt signaling pathway. Cell PhysioBiochem. 2016;38(3): 959-968.

39. Gentilini D, Busacca M, Di Francesco S, Vignali M, Vigano P, Di Blasio AM. PI3K/Akt and ERK1/2 signalling pathways are involved in endometrial cell migration induced by 17 beta-estradiol and growth factors. Mol HumReprod. 2007;13(5):317-322.

40. Fu Z, Zou F, Deng H, Zhou H, Liu L. Estrogen protects SGC7901 cells from endoplasmic reticulum stress-induced apoptosis by the Akt pathway. Oncol Lett. 2014;7(2):560-564.

41. Le AW, Shan L, Wang ZH, Dai XY, Xiao TH, Zuo R. Effects of icariin on the expression of ER, VEGF, and KDR in the endometrial cells of thin endometrium(J). Genetics and molecular research: GMR. 2015;14(3) $11250-11258$

\section{Dovepress}

Journal Citation Reports/Science Edition, EMBase, Scopus and the Elsevier Bibliographic databases. The manuscript management system is completely online and includes a very quick and fair peer-review system, which is all easy to use. Visit http://www.dovepress.com/ testimonials.php to read real quotes from published authors. 